EPJ Web of Conferences 7, 01002 (2010)

DOI:10.1051/epjconf/20100701002

(C) Owned by the authors, published by EDP Sciences, 2010

\title{
Color Glass Condensate and initial stages of heavy-ion collisions
}

François Gelis

IPhT, CEA/Saclay

\begin{abstract}
We introduce the concept of Color Glass Condensate, that describes the wave-function of a nucleon or nucleus at high energy. Then, we explain the relevance of this effective theory in the calculation of particle production in heavy ion collisions, and we show how it can be used in order to make predictions for the initial stages of these collisions - in particular in the task of providing initial conditions for hydrodynamical simulations.
\end{abstract}

\section{References}

1. F. Gelis, T. Lappi, R. Venugopalan, Int. J. Mod. Phys. E16, (2007) 2595-2637. F. Gelis, T. Lappi, R. Venugopalan, Int. J. Mod. Phys. E16, (2007) 2595-2637.

2. F. Gelis, R. Venugopalan, Acta Phys. Polon. B37, (2006) 3253-3314.

3. E. Iancu, R. Venugopalan, in Quark Gluon Plasma 3, Eds. R.C. Hwa and X.N.Wang, (World Scientific, 2003).

4. E. Iancu, A. Leonidov, L. McLerran, in Cargese 2001, QCD perspectives on hot and dense matter, (2001) 74145.

This is an Open Access article distributed under the terms of the Creative Commons Attribution-Noncommercial License 3.0, which permits unrestricted use, distribution, and reproduction in any noncommercial medium, provided the original work is properly cited. 


\section{Color Glass Condensate and and initial stages of heavy-ion collisions}

Dubna, July 2008

François Gelis IPhT, CEA/Saclay

Outline

(1) Introduction

(2) Color Glass Condensate

(3) Multi-gluon correlations in AA collisions

(4) Phenomenology

François Gelis

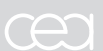

Introduction

Hydrodynamics

Correlations at large $\Delta Y$

CGC

Gluon saturation

Color Glass Condensate

AA collisions

Power counting

1-gluon spectrum at LO

Leading Log factorization

Multi-gluon correlations

Phenomenology

Glasma flux tubes

Gluon multiplicity 
(1) Introduction

Hydrodynamics

Correlations at large $\Delta Y$

(2) Color Glass Condensate

Gluon saturation

Color Glass Condensate

(3) Multi-gluon correlations in AA collisions

Power counting

Single gluon spectrum at LO

Leading Log factorization

Multi-gluon correlations

(4) Phenomenology

The ridge from glasma flux tubes

Gluon multiplicity

\section{Reminder on hydrodynamics}

Equations of hydrodynamics :

$$
\begin{aligned}
\partial_{\mu} T^{\mu \nu} & =0 \text { (energy-momentum conservation) } \\
\partial_{\mu} J_{\mathrm{B}}^{\mu} & =0 \text { (baryon number conservation) }
\end{aligned}
$$

- These equations contain only first order time derivatives

- Required initial conditions :

$$
T^{\mu \nu}\left(\tau=\tau_{0}, \eta, \overrightarrow{\boldsymbol{x}}_{\perp}\right), J_{\mathrm{B}}^{\mu}\left(\tau=\tau_{0}, \eta, \overrightarrow{\boldsymbol{x}}_{\perp}\right)
$$

\section{Additional inputs :}

$$
\begin{aligned}
\text { Equation of state: } & p=f(\epsilon) \\
\text { Transport coefficients: } & \eta, \zeta, \cdots
\end{aligned}
$$


Initial correlations and hydrodynamics

- The equations of hydrodynamics are non-linear. Therefore, solving hydro evolution for event averaged initial conditions is not the same as solving hydro event-by-event, and averaging observables at the end :

$$
\operatorname{HYDRO}\left[\left\langle T_{\text {init }}^{\mu \nu}\right\rangle\right] \neq\left\langle\operatorname{HYDRO}\left[T_{\text {init }}^{\mu \nu}\right]\right\rangle
$$

- To study hydrodynamics event by event, one needs an event generator for $T^{\mu \nu}\left(\tau_{0}, \eta, \overrightarrow{\boldsymbol{x}}_{\perp}\right)$

- To achieve this, it is not sufficient to know the average $\left\langle T^{\mu \nu}\left(\tau_{0}, \eta, \overrightarrow{\boldsymbol{x}}_{\perp}\right)\right\rangle$. We also need correlations :

$$
\begin{aligned}
& \left\langle T^{\mu_{1} \nu_{1}}\left(\tau_{0}, \eta_{1}, \overrightarrow{\boldsymbol{x}}_{1 \perp}\right) T^{\mu_{2} \nu_{2}}\left(\tau_{0}, \eta_{2}, \overrightarrow{\boldsymbol{x}}_{2 \perp}\right)\right\rangle \\
& \left\langle T^{\mu_{1} \nu_{1}}\left(\tau_{0}, \eta_{1}, \overrightarrow{\boldsymbol{x}}_{1 \perp}\right) T^{\mu_{2} \nu_{2}}\left(\tau_{0}, \eta_{2}, \overrightarrow{\boldsymbol{x}}_{2 \perp}\right) T^{\mu_{3} \nu_{3}}\left(\tau_{0}, \eta_{3}, \overrightarrow{\boldsymbol{x}}_{3 \perp}\right)\right\rangle \\
& \ldots
\end{aligned}
$$

Long range rapidity correlations probe early dynamics

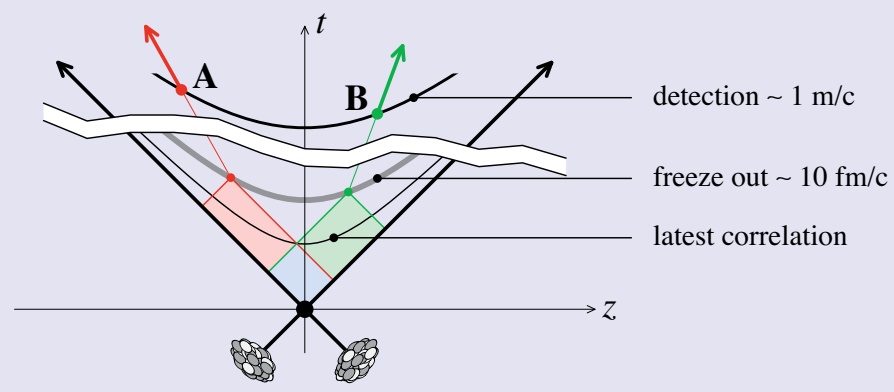

\section{Long range rapidity correlations are created early}

From causality, the latest time at which a correlation between two particles can be created is :

$$
t_{\text {correlation }} \leq t_{\text {freeze out }} e^{-\frac{1}{2}\left|y_{A}-y_{B}\right|}
$$

Example: $t_{\text {freeze out }}=10 \mathrm{fm} / \mathrm{c},\left|y_{A}-y_{B}\right|=6: t_{\text {correlation }} \leq 0.5 \mathrm{fm} / \mathrm{c}$ 
(1) Introduction

Hydrodynamics

Correlations at large $\Delta Y$

(2) Color Glass Condensate

Gluon saturation

Color Glass Condensate

(3) Multi-gluon correlations in AA collisions

Power counting

Single gluon spectrum at LO

Leading Log factorization

Multi-gluon correlations

(4. Phenomenology

The ridge from glasma flux tubes

Gluon multiplicity

\section{Gluon saturation}
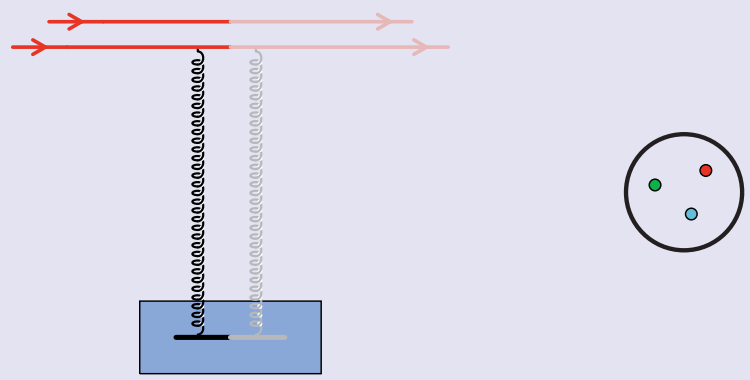

- consider a hadron or nucleus probed via gluon exchange

- at low energy, only valence quarks are present in the hadron wave function 
Gluon saturation

François Gelis

\section{Gluon saturation}
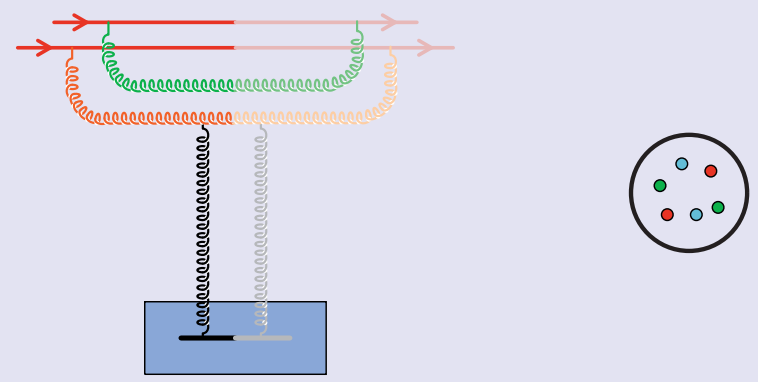

- when energy increases, new partons are emitted

- the emission probability is $\alpha_{s} \int \frac{d x}{x} \sim \alpha_{s} \ln \left(\frac{1}{x}\right)$, with $x$ the longitudinal momentum fraction of the gluon

- at small- $x$ (i.e. high energy), these logs need to be resummed

\section{Gluon saturation}
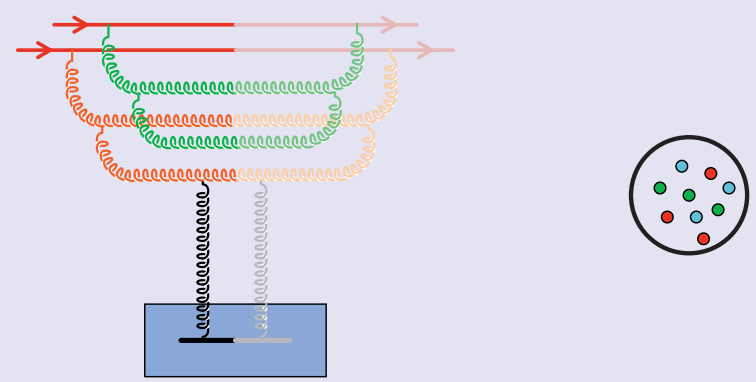

- as long as the density of constituents remains small, the evolution is linear: the number of partons produced at a given step is proportional to the number of partons at the previous step (BFKL)
François Gelis

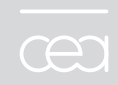

Introduction

Hydrodynamics

Correlations at large $\Delta Y$

CGC

Gluon saturation

Color Glass Condensate

AA collisions

Power counting

1-gluon spectrum at LO

Leading Log factorization

Multi-gluon correlations

Phenomenology

Glasma flux tubes

Gluon multiplicity 
Gluon saturation
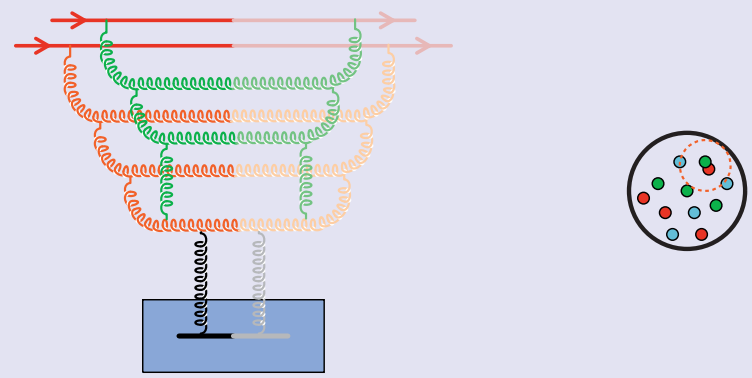

- eventually, the partons start overlapping in phase-space

- parton recombination becomes favorable

- after this point, the evolution is non-linear: the number of partons created at a given step depends non-linearly on the number of partons present previously

\section{Criterion for gluon recombination}

Gribov, Levin, Ryskin (1983)

\section{Number of gluons per unit area :}

$$
\rho \sim \frac{x G_{A}\left(x, Q^{2}\right)}{\pi R_{A}^{2}}
$$

\section{Recombination cross-section :}

$$
\sigma_{g g \rightarrow g} \sim \frac{\alpha_{s}}{Q^{2}}
$$

Recombination happens if $\rho \sigma_{g g \rightarrow g} \gtrsim 1$, i.e. $Q^{2} \lesssim Q_{s}^{2}$, with :

$$
Q_{S}^{2} \sim \frac{\alpha_{S} x G_{A}\left(x, Q_{S}^{2}\right)}{\pi R_{A}^{2}} \sim A^{1 / 3} \frac{1}{x^{0.3}}
$$

Note: At a given energy, the saturation scale is larger for a nucleus (for $A=200, A^{1 / 3} \approx 6$ ) 


\section{Saturation domain}

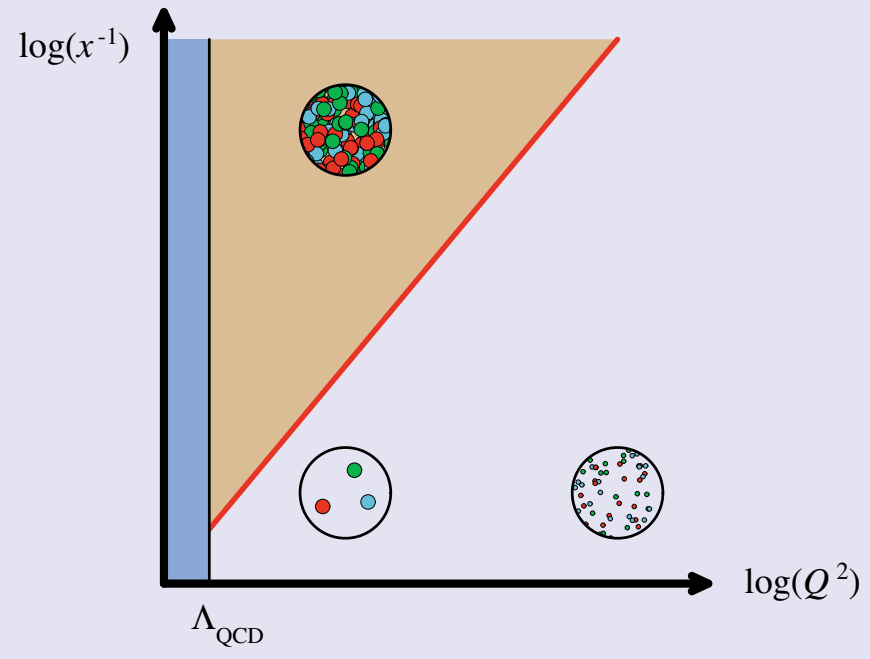

\section{Introduction}

Hydrodynamics

Correlations at large $\Delta Y$

CGC

\section{Gluon saturation}

Color Glass Condensate

AA collisions

Power counting

1-gluon spectrum at LO

Leading Log factorization

Multi-gluon correlations

Phenomenology

Glasma flux tubes

Gluon multiplicity

\section{Saturation domain}

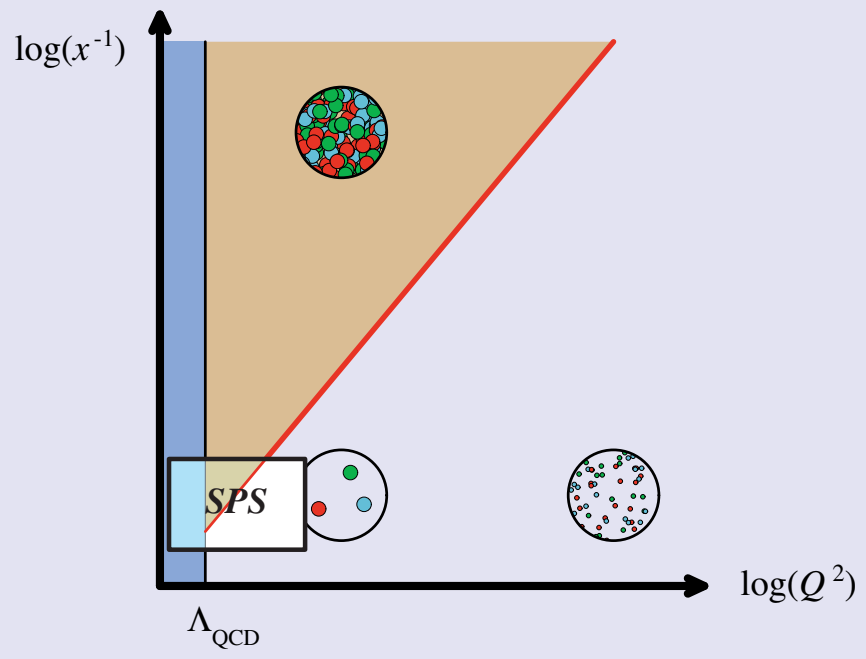

François Gelis

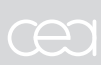

Introduction

Hydrodynamics

Correlations at large $\Delta Y$

CGC

Gluon saturation

Color Glass Condensate

AA collisions

Power counting

1-gluon spectrum at LO

Leading Log factorization

Multi-gluon correlations

Phenomenology

Glasma flux tubes

Gluon multiplicity 


\section{Saturation domain}

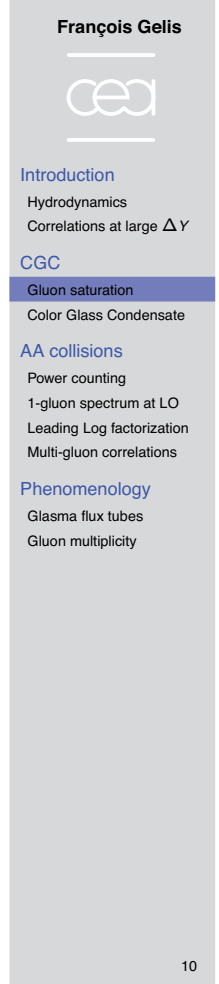

\section{Saturation domain}

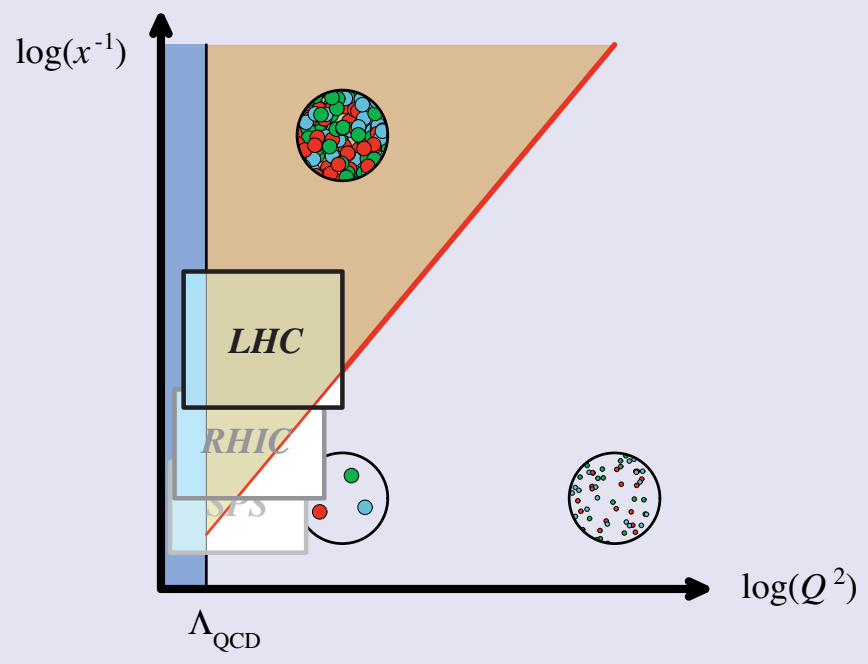

\section{François Gelis}

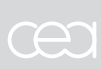

Introduction

Hydrodynamics

Correlations at large $\Delta Y$

CGC

Gluon saturation

Color Glass Condensate

AA collisions

Power counting

1-gluon spectrum at LO

Leading Log factorization

Multi-gluon correlations

Phenomenology

Glasma flux tubes

Gluon multiplicity 
Saturation domain
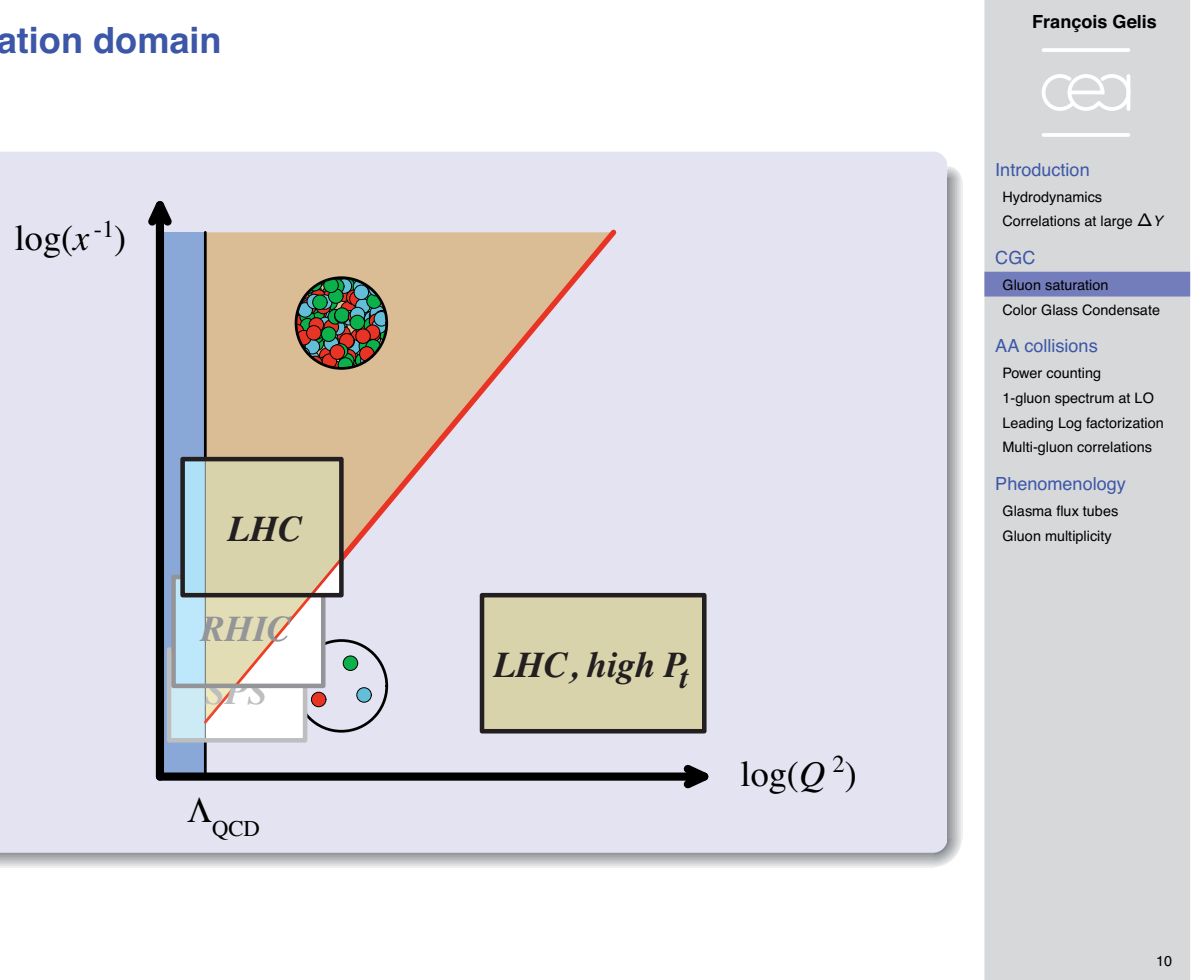

\section{CGC: Degrees of freedom}

$C G C=$ effective theory of small $x$ gluons

- The fast partons (large $x$ ) are frozen by time dilation $\triangleright$ described as static color sources on the light-cone :

$$
J^{\mu}=\delta^{\mu+} \delta\left(x^{-}\right) \rho\left(\overrightarrow{\boldsymbol{x}}_{\perp}\right) \quad\left(x^{-} \equiv(t-z) / \sqrt{2}\right)
$$

- Slow partons (small $x$ ) cannot be considered static over the time-scales of the collision process $\triangleright$ they must be treated as standard gauge fields

Eikonal coupling to the current $J^{\mu}: A_{\mu} J^{\mu}$

- The color sources $\rho$ are random, and described by a distribution functional $W_{Y}[\rho]$, with $Y$ the rapidity that separates "soft" and "hard"
François Gelis

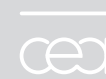

Introduction

Hydrodynamics

Correlations at large $\Delta Y$

CGC

Gluon saturation

Color Glass Condensate

AA collisions

Power counting

1-gluon spectrum at LO

Leading Log factorization

Multi-gluon correlations

Phenomenology

Glasma flux tubes

Gluon multiplicity 
CGC: renormalization group evolution

$$
\begin{aligned}
& \text { Evolution equation (JIMWLK) : } \\
& \qquad \frac{\partial W_{Y}}{\partial Y}=\mathcal{H} W_{Y} \\
& \qquad \mathcal{H}=\frac{1}{2} \int_{\overrightarrow{\boldsymbol{x}}_{\perp}, \overrightarrow{\boldsymbol{y}}_{\perp}} \frac{\delta}{\delta \widetilde{\mathcal{A}}^{+}\left(\epsilon, \overrightarrow{\boldsymbol{y}}_{\perp}\right)} \eta\left(\overrightarrow{\boldsymbol{x}}_{\perp}, \overrightarrow{\boldsymbol{y}}_{\perp}\right) \frac{\delta}{\delta \widetilde{\mathcal{A}}^{+}\left(\epsilon, \overrightarrow{\boldsymbol{x}}_{\perp}\right)}
\end{aligned}
$$

where $-\partial_{\perp}^{2} \widetilde{\mathcal{A}}^{+}\left(\epsilon, \overrightarrow{\boldsymbol{x}}_{\perp}\right)=\rho\left(\epsilon, \overrightarrow{\boldsymbol{x}}_{\perp}\right)$

- $\eta\left(\overrightarrow{\boldsymbol{x}}_{\perp}, \overrightarrow{\boldsymbol{y}}_{\perp}\right)$ is a non-linear functional of $\rho$

- This evolution equation resums all the powers of $\alpha_{s} \ln (1 / x)$ and of $Q_{s} / p_{\perp}$ that arise in loop corrections

- This equation simplifies into the BFKL equation when the source $\rho$ is small (one can expand $\eta$ in powers of $\rho$ )
1) Introduction

Hydrodynamics

Correlations at large $\Delta Y$

2. Color Glass Condensate

Gluon saturation

Color Glass Condensate

3 Multi-gluon correlations in AA collisions

Power counting

Single gluon spectrum at LO

Leading Log factorization

Multi-gluon correlations

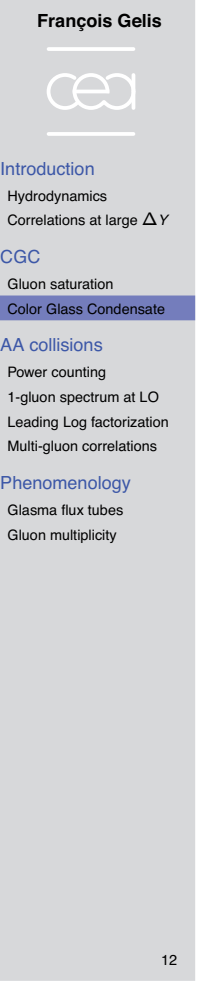

François Gelis

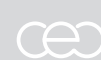

Introduction

Hydrodynamics

Correlations at large $\Delta Y$

CGC

Gluon saturation Color Glass Condensate

Power counting

1-gluon spectrum at LO

Leading Log factorization

Multi-gluon correlations

Phenomenology

Glasma flux tubes

Gluon multiplicity 


\section{Power counting}

$$
\begin{aligned}
& \boldsymbol{J}^{\mu} \equiv \underbrace{}_{\mathcal{S}_{Y M}} \underbrace{\delta^{\mu+} \rho_{1}\left(x^{-}, \overrightarrow{\boldsymbol{x}}_{\perp}\right)+\delta^{\mu-} \rho_{2}\left(x^{+}, \overrightarrow{\boldsymbol{x}}_{\perp}\right)} \\
& \mathcal{S}=\int d^{4} \boldsymbol{x} J^{\mu} A_{\mu}
\end{aligned}
$$

Hydrodynamics

Correlations at large $\Delta Y$

CGC

Gluon saturation

Color Glass Condensate

AA collisions

Power counting

1-gluon spectrum at LO

Leading Log factorization

Multi-gluon correlations

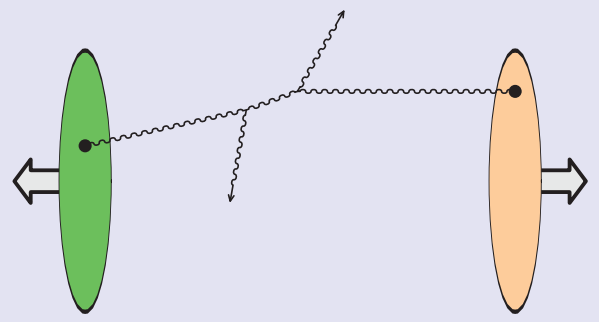

Phenomenology

Glasma flux tubes

Gluon multiplicity

- Dilute regime : one parton in each projectile interact

\section{Power counting}

$$
\begin{aligned}
& \boldsymbol{J}^{\mu} \equiv \delta^{\mu+} \rho_{1}\left(\boldsymbol{x}^{-}, \overrightarrow{\boldsymbol{x}}_{\perp}\right)+\delta^{\mu-} \rho_{2}\left(\boldsymbol{x}^{+}, \overrightarrow{\boldsymbol{x}}_{\perp}\right) \\
& \mathcal{S}=\underbrace{-\frac{1}{2} \int d^{4} X \operatorname{tr} F_{\mu \nu} F^{\mu \nu}}_{\mathcal{S}_{Y M}}+\int d^{4} X J^{\mu} A_{\mu}
\end{aligned}
$$

François Gelis

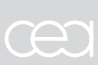

Introduction

Hydrodynamics

Correlations at large $\Delta Y$

CGC

Gluon saturation

Color Glass Condensate

AA collisions

\section{Power counting}

1-gluon spectrum at $L O$ Leading Log factorization Multi-gluon correlations

Phenomenology

Glasma flux tubes

Gluon multiplicity

- Dilute regime : one parton in each projectile interact

- Dense regime : multiparton processes become crucial 


\section{Power counting}

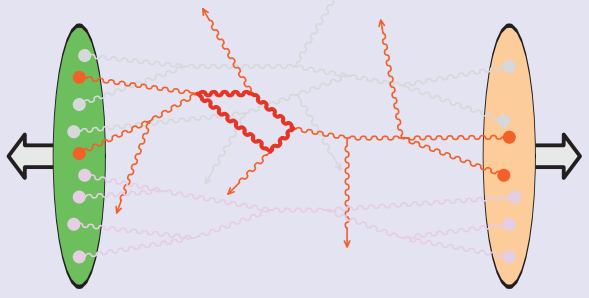

- In the saturated regime, the sources are of order $1 / \mathrm{g}$ (because $\langle\rho \rho\rangle \sim$ occupation number $\sim 1 / \alpha_{s}$ )

- Order of a connected diagram :

$$
\frac{1}{g^{2}} g^{\# \text { produced gluons }} g^{2 \text { (\# loops) }}
$$

Diagrammatic expansion of $d N_{1} / d^{3} p$

- The single inclusive spectrum has a simple diagrammatic representation :

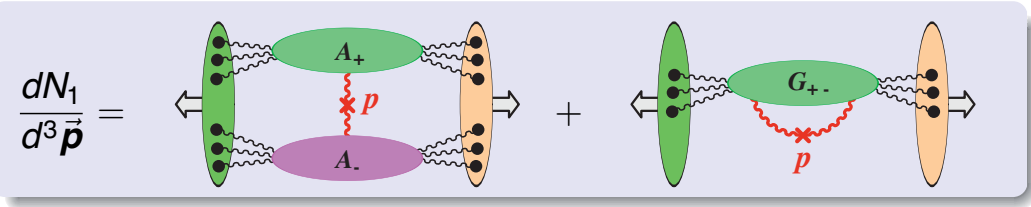

François Gelis

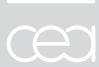

Gluon saturation

Color Glass Condensate

AA collisions

Power counting

1-gluon spectrum at LO

Leading Log factorization Multi-gluon correlations

Phenomenology

- There are only connected graphs (AGK cancellation)

- Perturbative expansion in the saturated regime :

$$
\frac{d N_{1}}{d^{3} \vec{p}}=\frac{1}{g^{2}}[\underbrace{c_{0}}_{\mathrm{LO}}+\underbrace{c_{1} g^{2}}_{\mathrm{NLO}}+\underbrace{c_{2} g^{4}}_{\mathrm{NNLO}}+\cdots]
$$


Expression in terms of classical fields at LO

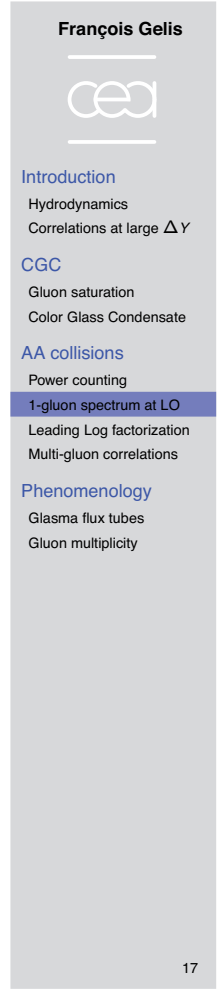

- $\mathcal{A}$ obeys the classical EOM : $\frac{\delta \mathcal{S}_{Y M}}{\delta \mathcal{A}}+J=0$

- The boundary conditions are very simple:

$$
\lim _{x^{0} \rightarrow-\infty} \mathcal{A}(x)=0
$$

\section{Initial classical fields}

\section{Lappi, McLerran (2006)}

- Immediately after the collision, the chromo- $\overrightarrow{\boldsymbol{E}}$ and $\overrightarrow{\boldsymbol{B}}$ fields are purely longitudinal :

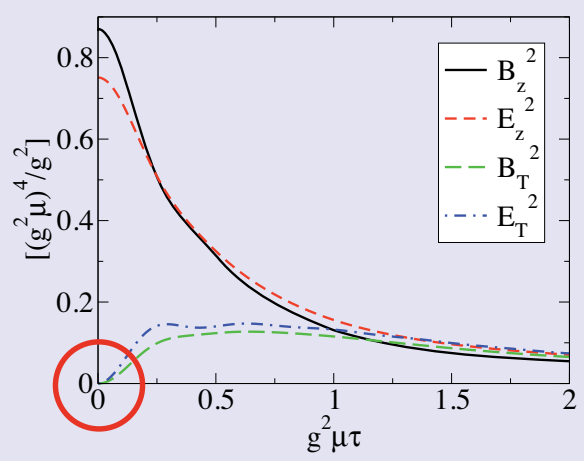


Initial classical fields

- The initial chromo- $\overrightarrow{\boldsymbol{E}}$ and $\overrightarrow{\boldsymbol{B}}$ fields form longitudinal "flux tubes" extending between the projectiles:

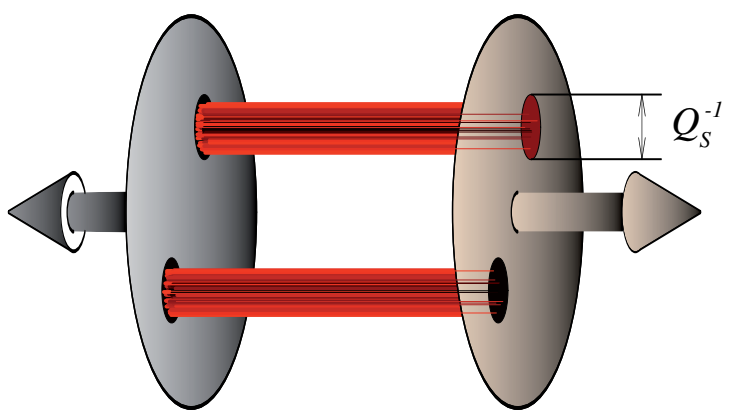

- The color correlation length in the transverse plane is $Q_{s}^{-1}$ $\triangleright$ flux tubes of diameter $Q_{s}^{-1}$, filling up the transverse area

\section{Single gluon spectrum at LO}

Krasnitz, Nara, Venugopalan (1999 - 2001), Lappi (2003)

- No analytic solution for the Yang-Mills equations, but straightforward numerically

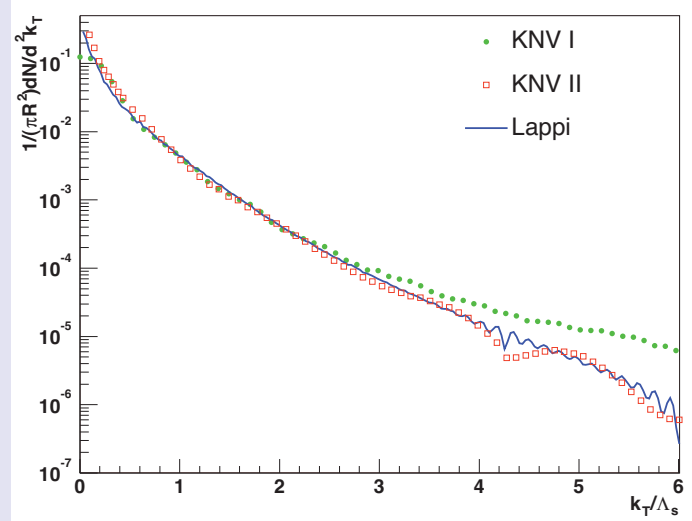




\section{What is factorization ?}

- The naive perturbative expansion of $d N_{1} / d^{3} \overrightarrow{\boldsymbol{p}}$,

$$
\frac{d N}{d^{3} \overrightarrow{\boldsymbol{p}}}=\frac{1}{g^{2}}\left[c_{0}+c_{1} g^{2}+c_{2} g^{4}+\cdots\right],
$$

assumes that the coefficients $c_{n}$ are of order one

- This assumption is upset by large logarithms of $1 / x_{1,2}$ :

$$
\begin{array}{rr}
c_{1}= & d_{10}+d_{20}+d_{21} \ln \left(\frac{1}{x_{1,2}}\right)+\underbrace{d_{11} \ln \left(\frac{1}{x_{1,2}}\right)}_{\text {Leading Log terms }} \\
c_{2}=d_{22} \ln ^{2}\left(\frac{1}{x_{1,2}}\right)
\end{array}
$$

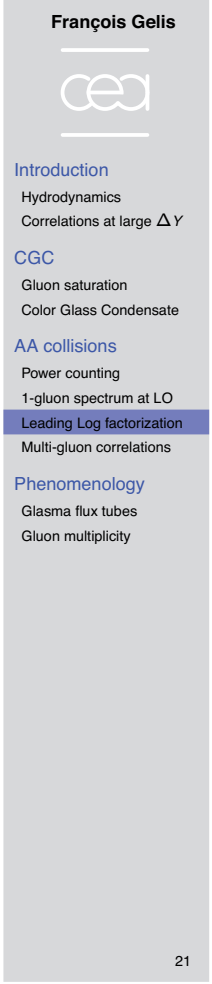

- Factorizability: the logarithms must be universal and resummable into functionals that depend only on the projectiles being collided

Why factorization works: causality

$$
\tau_{\mathrm{coll}} \sim \boldsymbol{E}^{-1}
$$
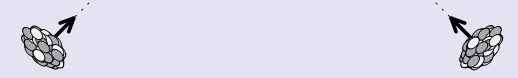

- The duration of the collision is very short: $\tau_{\text {coll }} \sim E^{-1}$
François Gelis

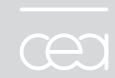

Introduction

Hydrodynamics

Correlations at large $\Delta Y$

CGC

Gluon saturation

Color Glass Condensate

AA collisions

Power counting

1-gluon spectrum at LO

Leading Log factorization

Multi-gluon correlations

Phenomenology 
Why factorization works: causality

$$
\bullet \quad \tau_{\text {coll }} \sim E^{-1}
$$
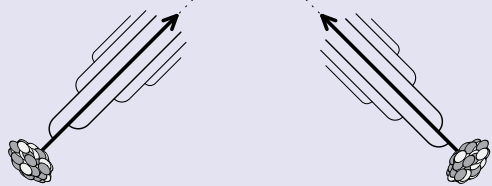

- The duration of the collision is very short: $\tau_{\text {coll }} \sim E^{-1}$

- The logarithms we want to resum arise from the radiation of soft gluons, which takes a long time $\triangleright$ it must happen (long) before the collision

Why factorization works: causality

$$
\bullet \tau_{\text {coll }} \sim \boldsymbol{E}^{-1}
$$

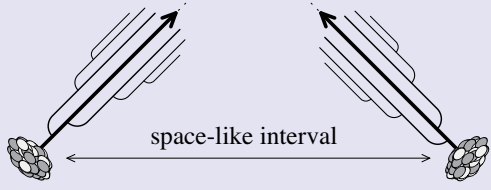

- The duration of the collision is very short: $\tau_{\text {coll }} \sim E^{-1}$

- The logarithms we want to resum arise from the radiation of soft gluons, which takes a long time $\triangleright$ it must happen (long) before the collision

- The projectiles are not in causal contact before the impact $\triangleright$ the logarithms are intrinsic properties of the projectiles, independent of the measured observable

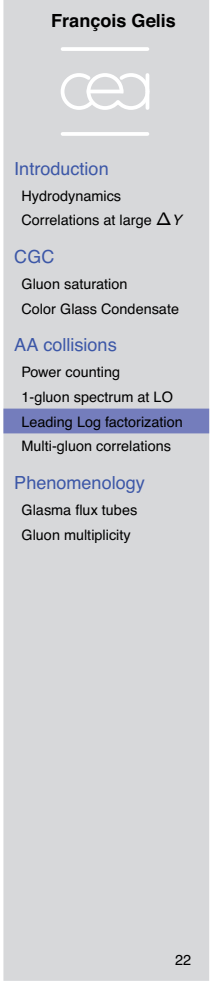

\footnotetext{
François Gelis

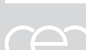

Introduction

Hydrodynamics

Correlations at large $\Delta Y$

CGC

Gluon saturation

Color Glass Condensate

AA collisions

Power counting

1-gluon spectrum at LO

Leading Log factorizatio

Multi-gluon correlations

Phenomenology 
Why the proof is complicated: strong fields

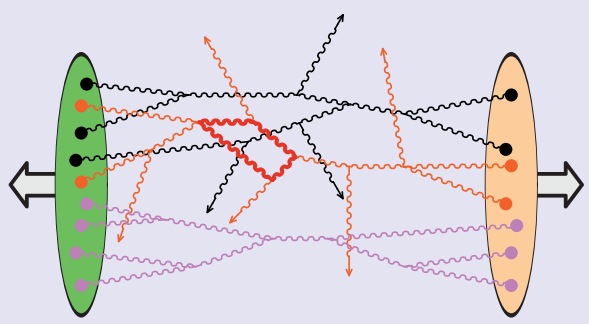

- Procedure: (i) calculate the 1-loop corrections, (ii) disentangle the logarithms from the finite contributions, (iii) show that the logs can be assigned to the projectiles

- Problem: strong fields, analytic calculation not feasible

$\triangleright$ Take advantage of the retarded nature of the boundary conditions in order to separate the initial state evolution (calculable analytically) from the collision itself (hopeless)

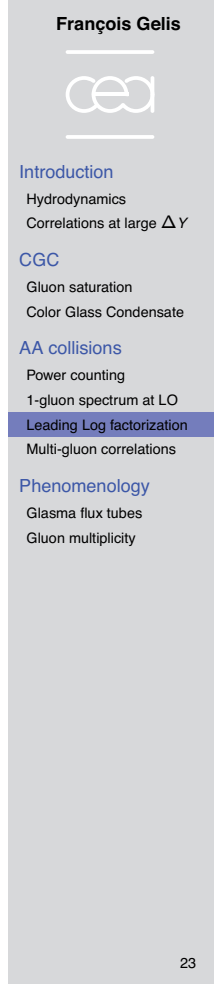

Factorization in two steps: FG, Lappi, Venugopalan (2008)

: The NLO gluon spectrum can be written as a perturbation of the initial value of the classical fields on the light-cone :

$$
\left.\frac{d N_{1}}{d^{3} \overrightarrow{\boldsymbol{p}}}\right|_{\mathrm{NLO}}=\left.\left[\frac{1}{2} \int_{\vec{u}, \vec{v} \in \mathrm{LC}} \mathcal{G}(\vec{u}, \vec{v}) \mathbb{T}_{u} \mathbb{T}_{\boldsymbol{v}}+\int_{\vec{u} \in \mathrm{LC}} \beta(\vec{u}) \mathbb{T}_{\boldsymbol{u}}\right] \frac{d N_{1}}{d^{3} \overrightarrow{\boldsymbol{p}}}\right|_{\mathrm{LO}}
$$

$$
\left(\mathbb{T}_{\boldsymbol{u}} \sim \delta / \delta \mathcal{A}_{\text {initial }}(\boldsymbol{u}) \quad, \quad \mathcal{G}, \beta \text { are calculable analytically }\right)
$$

II : The operator $[\cdots]$ is related to the JIMWLK Hamiltonian:

$\frac{1}{2} \int \mathcal{G}(\vec{u}, \vec{v}) \mathbb{T}_{u} \mathbb{T}_{\boldsymbol{v}}+\int \beta(\vec{u}) \mathbb{T}_{\boldsymbol{u}}=\log \left(\frac{\Lambda^{+}}{p^{+}}\right) \times \mathcal{H}_{1}+\log \left(\frac{\Lambda^{-}}{p^{-}}\right) \times \mathcal{H}_{2}$

$\vec{u}, \vec{v} \in \mathrm{LC} \quad+$ finite terms

$\triangleright$ Factorization follows easily
François Gelis

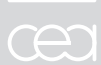

Introduction

Hydrodynamics

Correlations at large $\Delta Y$

CGC

Gluon saturation

Color Glass Condensate

AA collisions

Power counting

1-gluon spectrum at LO

Leading Log factorizatio

Multi-gluon correlations

Phenomenology

Glasma flux tubes

Gluon multiplicity 
Leading Log factorization

- By averaging over all the configurations of the sources in the two projectiles, we get a factorized formula for the resummation of the leading log terms to all orders :

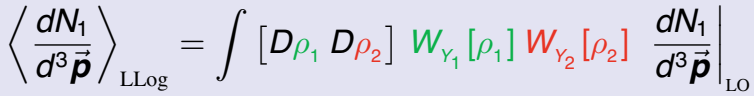

$$
\begin{aligned}
& \text { with : } \frac{\partial}{\partial Y} W_{Y}=\mathcal{H} W, \quad Y_{1}=\log \left(\sqrt{s} / p^{+}\right), \quad Y_{2}=\log \left(\sqrt{s} / p^{-}\right)
\end{aligned}
$$

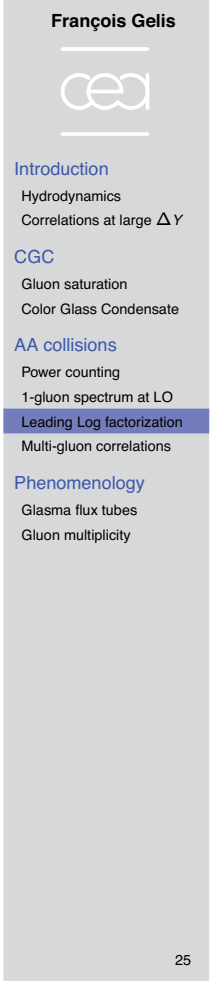

- The distributions $W\left[\rho_{1,2}\right]$ must be evolved up to the rapidity of the produced gluon

\section{Multigluon spectrum at LO}

\section{FG, Lappi, Venugopalan (2008)}

- In the saturated regime, the inclusive $n$-gluon spectrum at Leading Order is the product of $n 1$-gluon spectra:

$$
\left.\frac{d N_{n}}{d^{3} \overrightarrow{\boldsymbol{p}}_{1} \cdots d^{3} \overrightarrow{\boldsymbol{p}}_{n}}\right|_{\text {LO }}=\left.\frac{d N_{1}}{d^{3} \overrightarrow{\boldsymbol{p}}_{1}}\right|_{\text {LO }} \times \cdots \times\left.\frac{d N_{1}}{d^{3} \overrightarrow{\boldsymbol{p}}_{n}}\right|_{\text {LO }}
$$

- At LO, in a given configuration of the sources $\rho_{1,2}$, the $n$ gluons are not correlated

- Note: this is true for the bulk $\left(p_{\perp} \lesssim Q_{S}\right)$, but not for the tail of the distribution
François Gelis

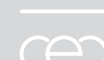

Introduction

Hydrodynamics

Correlations at large $\Delta Y$

CGC

Gluon saturation

Color Glass Condensate

AA collisions

Power counting

1-gluon spectrum at LO

Leading Log factorization

Phenomenology
Multi-gluon correlations 
Multigluon spectrum at NLO

- At NLO, one has again:

$$
\begin{aligned}
& \left.\frac{d N_{n}}{d^{3} \overrightarrow{\boldsymbol{p}}_{1} \cdots d^{3} \overrightarrow{\boldsymbol{p}}_{n}}\right|_{\mathrm{NLO}}= \\
& \quad=\left.\left[\frac{1}{2} \int_{\overrightarrow{\boldsymbol{u}}, \overrightarrow{\boldsymbol{v}} \in \mathrm{LC}} \mathcal{G}(\vec{u}, \overrightarrow{\boldsymbol{v}}) \mathbb{T}_{\boldsymbol{u}} \mathbb{T}_{\boldsymbol{v}}+\int_{\overrightarrow{\boldsymbol{u}} \in \mathrm{LC}} \beta(\vec{u}) \mathbb{T}_{\boldsymbol{u}}\right] \frac{d N_{n}}{d^{3} \overrightarrow{\boldsymbol{p}}_{1} \cdots d^{3} \overrightarrow{\boldsymbol{p}}_{n}}\right|_{\mathrm{LO}}
\end{aligned}
$$

François Gelis

00

troduction

Hydrodynamics

Correlations at large $\Delta Y$

CGC

Gluon saturation

Color Glass Condensate

AA collisions

Power counting

1-gluon spectrum at LO

Leading Log factorization

Multi-gluon correlations

Phenomenology

Glasma flux tubes

Gluon multiplicity

- Correlations appear at NLO thanks to the operator $\mathcal{G}(\vec{u}, \vec{v}) \mathbb{T}_{\boldsymbol{u}} \mathbb{T}_{v}$, which can link two different gluons

- Thanks to their universal structure, we can factorize these correlations into the distributions $W\left[\rho_{1,2}\right]$

\section{Leading Log factorization}

\section{Factorization formula for the $n$-gluon spectrum}

$$
\begin{aligned}
\left\langle\frac{d N_{n}}{d^{3} \overrightarrow{\boldsymbol{p}}_{1} \cdots d^{3} \overrightarrow{\boldsymbol{p}}_{n}}\right\rangle_{\text {LLog }} & =\int\left[D_{\rho_{1}} \boldsymbol{D}_{\rho_{2}}\right] W\left[\rho_{1}\right] W\left[\rho_{2}\right] \\
& \times\left.\frac{d N_{1}}{d^{3} \overrightarrow{\boldsymbol{p}}_{1}}\right|_{\text {LO }} \times \cdots \times\left.\frac{d N_{1}}{d^{3} \overrightarrow{\boldsymbol{p}}_{n}}\right|_{\text {LO }}
\end{aligned}
$$

- This formula tells us that (in the Leading Log approximation) all the correlations arise from the $W[\rho]$ 's $\triangleright$ they pre-exist in the wave-function of the projectiles

- Note: some short range correlations will also arise from splittings in the final state (not taken into account here, because does not come with a $\ln (s)$ )
François Gelis

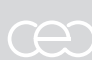

Introduction

Hydrodynamics

Correlations at large $\Delta Y$

CGC

Gluon saturation

Color Glass Condensate

AA collisions

Power counting

1-gluon spectrum at $L O$

Leading Log factorization Multi-gluon correlations

Phenomenology

Glasma flux tubes

Gluon multiplicity 
(1) Introduction

Hydrodynamics

Correlations at large $\Delta Y$

(2) Color Glass Condensate

Gluon saturation

Color Glass Condensate

(3) Multil-gluon correlations in AA collisions

Power counting

Single gluon spectrum at LO

Leading Log factorization

Multi-gluon correlations

(4) Phenomenology

The ridge from glasma flux tubes

Gluon multiplicity

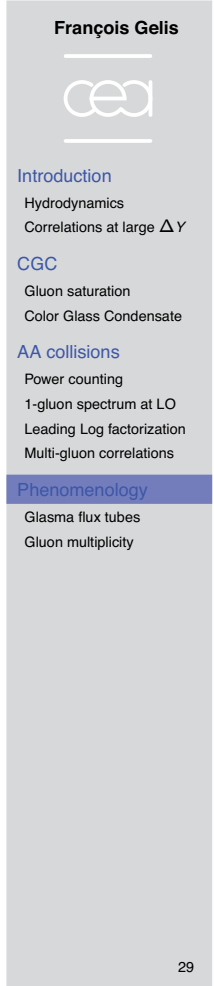

\section{2-hadron correlations at RHIC}

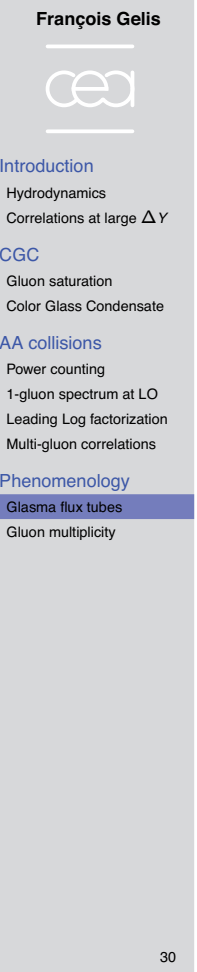

- Long range correlation in $\Delta \eta$ (rapidity)

- Narrow correlation in $\Delta \varphi$ (azimuthal angle) 
Causality constraint

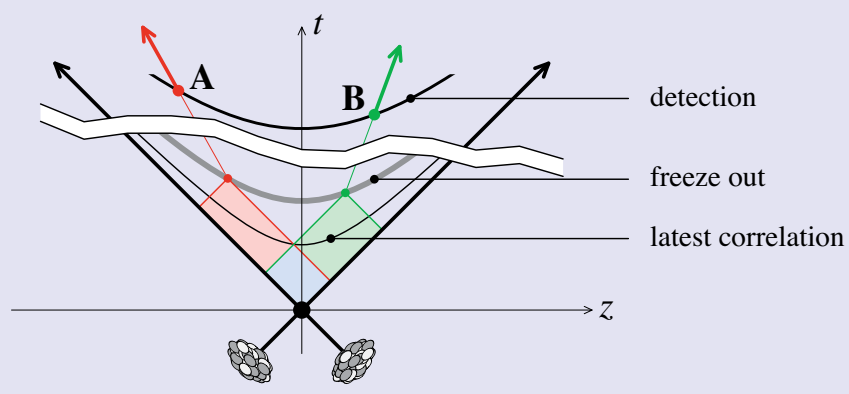

Introduction

Hydrodynamics

Correlations at large $\Delta Y$

CGC

Gluon saturation

Color Glass Condensate

AA collisions

Power counting

1-gluon spectrum at LO

Leading Log factorization

Multi-gluon correlations

Phenomenology

Glasma flux tubes

Gluon multiplicity

\section{Long range rapidity correlations are created early}

From causality, the latest time at which a correlation between two particles can be created is :

$$
t_{\text {correlation }} \leq t_{\text {freeze out }} e^{-\frac{1}{2}\left|y_{A}-y_{B}\right|}
$$

\section{Glasma flux tubes}

Dumitru, FG, McLerran, Venugopalan (2008)

Gavin, McLerran, Moschelli (2008)

Dusling, Fernandez-Fraile, Venugopalan (2009)

- Was there something independent of $\eta$ at early times? $\triangleright$ the chromo- $\vec{E}$ and $\vec{B}$ fields produced in the collision

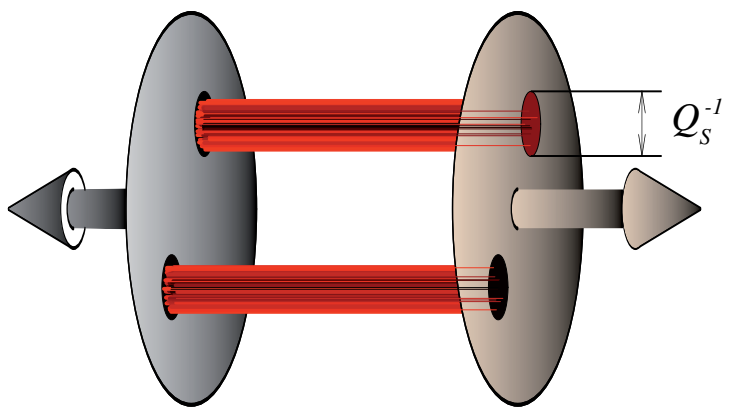

- The color correlation length in the transverse plane is $Q_{s}^{-1}$ $\triangleright$ flux tubes of diameter $Q_{S}^{-1}$, filling up the transverse area 
Glasma flux tubes

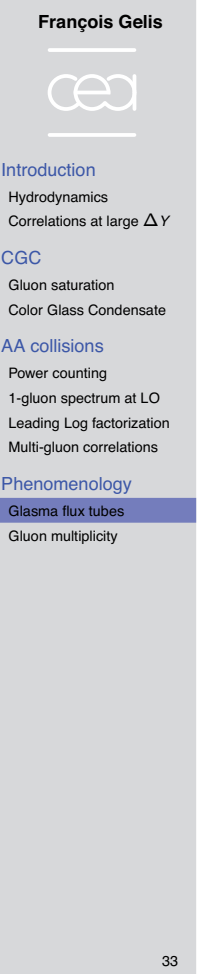

- $\eta$-independent fields lead to long range correlations in the 2-particle spectrum :

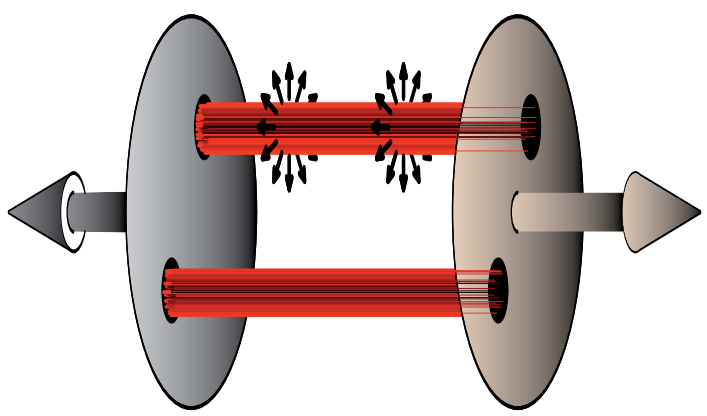

- $\eta$-independent fields lead to long range correlations in the 2-particle spectrum :

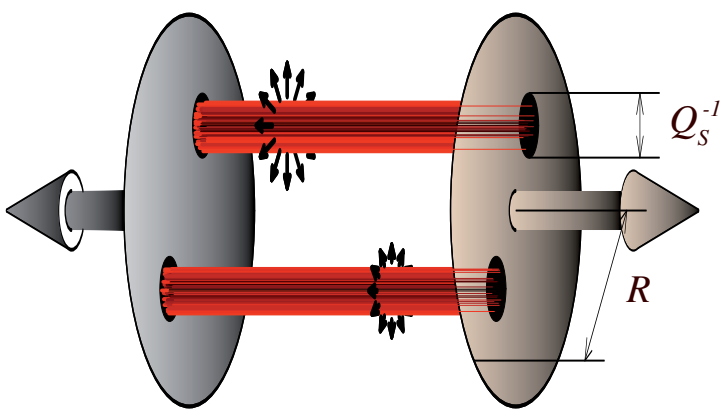

François Gelis

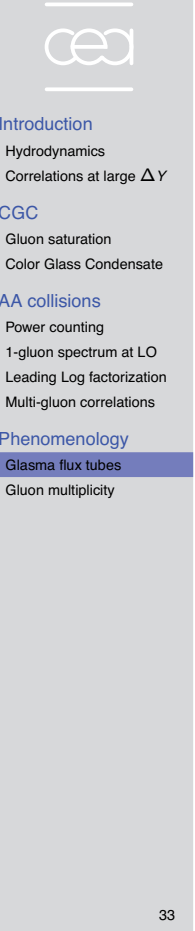

- Particles emitted by different flux tubes are not correlated $\triangleright\left(R Q_{S}\right)^{-2}$ sets the strength of the correlation 
- $\eta$-independent fields lead to long range correlations in the 2-particle spectrum :

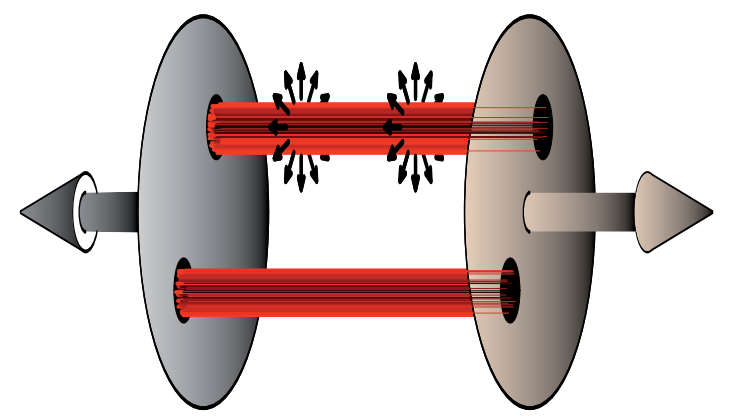

Gluon saturation

Color Glass Condensate

AA collisions

Power counting

1-gluon spectrum at LO

Leading Log factorization

Multi-gluon correlations

Phenomenology

Glasma flux tubes

Gluon multiplicity

- Particles emitted by different flux tubes are not correlated $\triangleright \quad\left(R Q_{S}\right)^{-2}$ sets the strength of the correlation

- At early times, the correlation is flat in $\Delta \varphi$

\section{Glasma flux tubes}

- $\eta$-independent fields lead to long range correlations in the 2-particle spectrum :

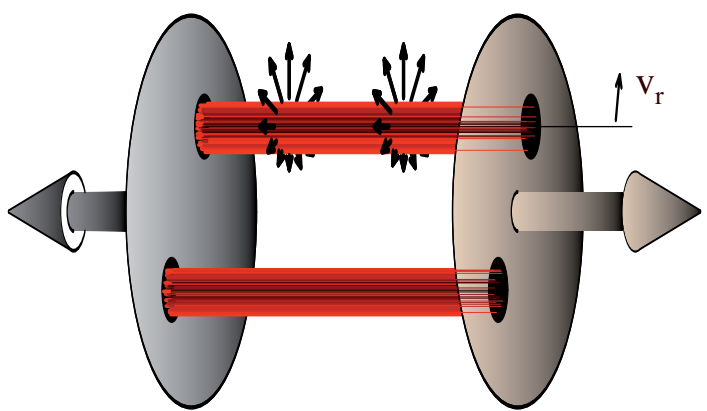

- Particles emitted by different flux tubes are not correlated $\triangleright \quad\left(R Q_{s}\right)^{-2}$ sets the strength of the correlation

- At early times, the correlation is flat in $\Delta \varphi$ A collimation in $\Delta \varphi$ is produced later by radial flow 


\section{Gluon multiplicity}

FG, Lappi, McLerran (2009)

- The combinatorics of color source averages in a single glasma flux tube leads to:

$$
\langle N(N-1) \cdots(N-p+1)\rangle-\text { disc. terms }=(p-1) !\langle N\rangle^{p}
$$

- Bose-Einstein distribution

- If one superimposes $k$ such flux tubes emitting independently:

$$
\langle N(N-1) \cdots(N-p+1)\rangle-\text { disc. terms }=(p-1) !\left[\frac{\langle N\rangle}{k}\right]^{p}
$$

- Negative binomial distribution with parameters $\langle N\rangle, k$

- $k$ is the number of flux tubes: $k \sim Q_{s}^{2} R^{2} \sim$ \# participants

- Experimentally: it seems to work... 